\title{
A next-generation ground array for the detection of ultrahigh-energy cosmic rays: the Fluorescence detector Array of Single-pixel Telescopes (FAST)
}

\author{
Toshihiro Fujii ${ }^{1, *}$, Max Malacari ${ }^{2}$, Justin Albury ${ }^{3}$, Jose A. Bellido ${ }^{3}$, Ladislav Chytka ${ }^{4}$, John Farmer $^{2}$, Petr Hamal $^{4}$, Pavel \\ Horvath $^{4}$, Miroslav Hrabovsky ${ }^{4}$, Dusan Mandat ${ }^{5}$, John N. Matthews ${ }^{6}$, Xiaochen $\mathrm{Ni}^{2}$, Libor Nozka ${ }^{5}$, Miroslav Palatka ${ }^{5}$, \\ Miroslav Pech ${ }^{5}$, Paolo Privitera ${ }^{2}$, Petr Schovanek ${ }^{5}$, Stan B. Thomas ${ }^{6}$, and Petr Travnicek ${ }^{5}$ \\ ${ }^{1}$ Institute for Cosmic Ray Research, University of Tokyo, Kashiwa, Chiba, Japan \\ ${ }^{2}$ Kavli Institute for Cosmological Physics, University of Chicago, Chicago, IL, USA \\ ${ }^{3}$ Department of Physics, University of Adelaide, Adelaide, S.A., Australia \\ ${ }^{4}$ Palacky University, RCPTM, Olomouc, Czech Republic \\ ${ }^{5}$ Institute of Physics of the Academy of Sciences of the Czech Republic, Prague, Czech Republic \\ ${ }^{6}$ High Energy Astrophysics Institute and Department of Physics and Astronomy, University of Utah, Salt Lake City, UT, USA
}

\begin{abstract}
The origin and nature of ultrahigh-energy cosmic rays (UHECRs) is one of the most intriguing and important mysteries in astroparticle physics. The two largest observatories currently in operation, the Telescope Array Experiment in central Utah, USA, and the Pierre Auger Observatory in western Argentina, have been steadily observing UHECRs in both hemispheres for over a decade. We highlight the latest results from both of these experiments, and address the requirements for a next-generation UHECR observatory. The Fluorescence detector Array of Single-pixel Telescopes (FAST) is a design concept for a next-generation UHECR observatory, addressing the requirements for a large-area, low-cost detector suitable for measuring the properties of the highest energy cosmic rays with an unprecedented aperture. We have developed a full-scale prototype consisting of four $200 \mathrm{~mm}$ photomultiplier-tubes at the focus of a segmented mirror of $1.6 \mathrm{~m}$ in diameter. Over the last three years, we installed three such prototypes at the Black Rock Mesa site of the Telescope Array Experiment. These telescopes have been steadily taking data since installation. We report on preliminary results of the full-scale FAST prototypes, including measurements of distant ultraviolet lasers and UHECRs. Futhermore, we discuss our plan to install an additional identical FAST prototype at the Pierre Auger Observatory. Possible benefits to the Telescope Array and the Pierre Auger Observatory include a comparison of the transparency of the atmosphere above both experiments, a study of the systematic uncertainty associated with their existing fluorescence detectors, and a cross-calibration of their energy and $X_{\max }$ scales.
\end{abstract}

\section{Ultrahigh-energy cosmic rays}

Since ultrahigh-energy cosmic rays (UHECRs) are the most energetic particles in the universe, their origins are ostensibly related to extremely energetic astrophysical phenomena, such as gamma-ray bursts, active galactic nuclei, or other exotic processes such as the decay or annihilation of super-heavy relic particles created in an early phase of the development of the universe [1]. However, their origin and acceleration mechanism above $10^{20} \mathrm{eV}$ are still unknown. Therefore, they are one of the most intriguing and important mysteries in particle astrophysics and astronomy.

Following the detection of a cosmic-ray event with an energy of $10^{20} \mathrm{eV}$ by J. Linsley [2] in 1963, K. Greisen, G. T. Zatsepin and V. A. Kuzmin predicted the UHECR energy spectrum to be suppressed above $10^{19.7} \mathrm{eV}$ due to the interaction of high-energy particles with the $3 \mathrm{~K}$ cosmic microwave background radiation via pion production, the so-called GZK cutoff $[3,4]$. If the GZK cutoff

*e-mail: fujii@cr.scphys.kyoto-u.ac.jp, Now at the Hakubi Center for Advanced Research and Graduate School of Science, Kyoto University exists, the origin of UHECRs is significantly restricted to nearby sources distributed non-uniformly within 50$100 \mathrm{Mpc}$. Additionally, UHECRs propagate with less deflection by magnetic fields due to their enormous kinetic energies. As a result, the arrival directions of UHECRs should be correlated with the directions of extremely energetic sources or objects, leading to the possibility of nextgeneration particle astronomy with UHECRs, illuminating extremely energetic phenomena in the nearby universe.

Given the minute flux of UHECRs, less than one particle per century per square kilometer at the highest energies, a very large area must be instrumented in order to collect significant statistics. The energy, arrival direction, and mass composition of UHECRs can be inferred from studies of the cascades of secondary particles (Extensive Air Shower, EAS) produced by their interaction with the Earth's atmosphere.

Two well-established techniques are used for UHECR detection: arrays of detectors (e.g. plastic scintillators or water-Cherenkov stations) sample EAS particles reaching the ground, and large-field-of-view telescopes allow 


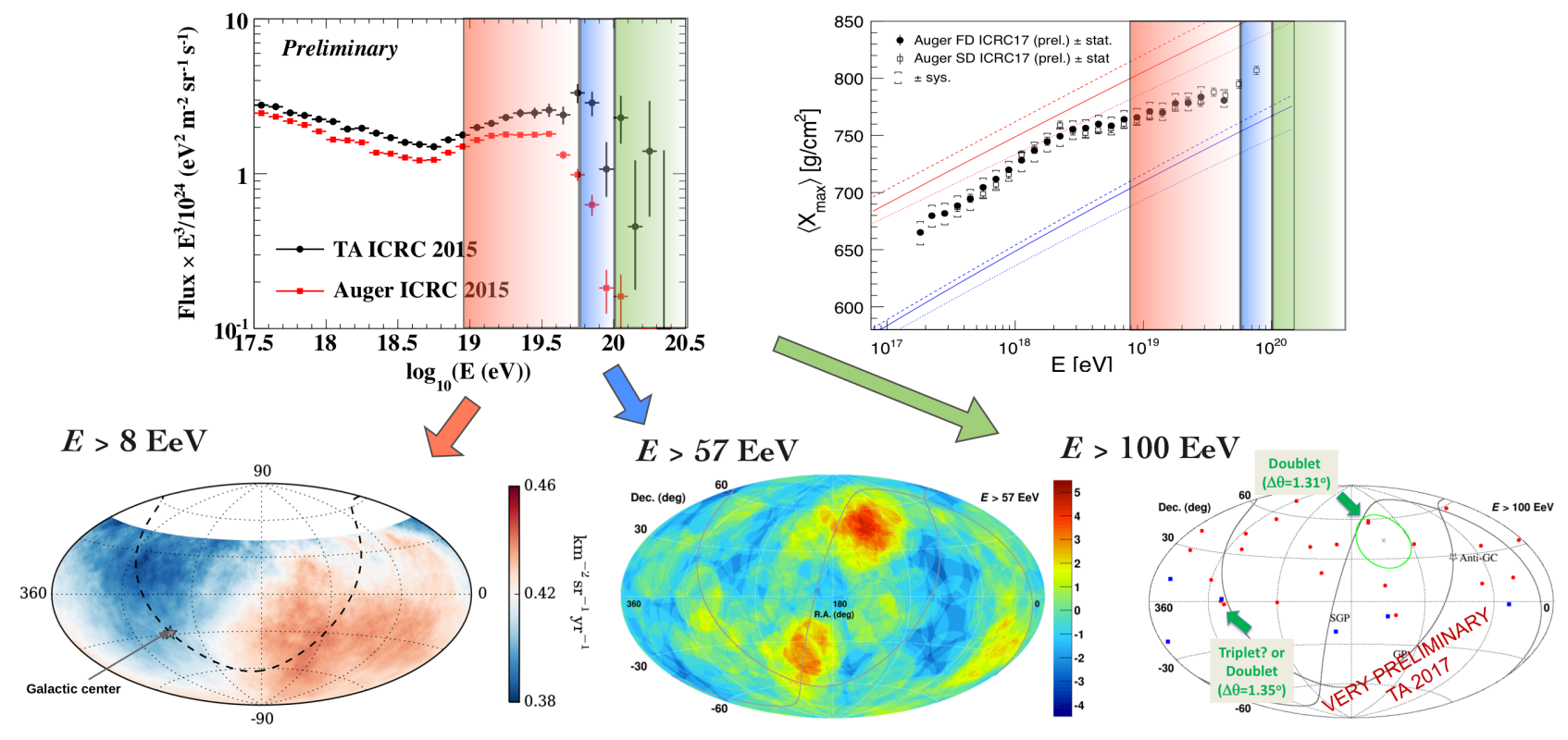

Figure 1. Overview of three important observables reported by the Pierre Auger Observatory and Telescope Array Experiment: energy spectrum [5-7] mass composition [8-10] and arrival direction [11-14] with energy ranges indicated above: 8 EeV (red), 57 EeV (blue) and $100 \mathrm{EeV}$ (green).

for reconstruction of the shower development in the atmosphere by imaging ultraviolet fluorescence light from atmospheric nitrogen excited by EAS particles. Two giant observatories, one in each hemisphere, the Pierre Auger Observatory (Auger) in Mendoza, Argentina [15] and the Telescope Array Experiment (TA) in Utah, USA [16, 17], combine the two techniques with arrays of particle detectors overlooked by fluorescence detectors (FD).

\section{Latest results from current UHECR observatories}

Figure 1 shows the latest results on the energy spectrum, arrival directions and mass composition of UHECRs reported by the Pierre Auger Observatory and the Telescope Array Experiment [5-14]. Both observatories have measured a dip around $10^{18.7} \mathrm{eV}$ and a suppression above $10^{19.7} \mathrm{eV}[5,6]$. The suppression is consistent with expectation from the GZK cutoff, however the shape of the suppression is discrepant between the two measurements [7].

The mass composition reported by Auger through $X_{\max }$ (the depth in the atmosphere at which the EAS reaches its maximum energy deposit) suggests a transition from light nuclei at around $10^{18.3} \mathrm{eV}$ to heavier nuclei up to energies of $10^{19.6} \mathrm{eV}[8,9]$. In addition, the $X_{\max }$ distributions measured by Auger and TA below $10^{19} \mathrm{eV}$ are consistent within systematic uncertainties [19, 20]. Recently Auger reported a new mass composition result using the surface detector array, sensitive to primary energies above $57 \mathrm{EeV}$, using measurements of the signal rise time [10]. However, there is currently no mass composition result above $100 \mathrm{EeV}$.

Figure 1 also includes the measured arrival directions of UHECRs above $8 \mathrm{EeV}, 57 \mathrm{EeV}$ and $100 \mathrm{EeV}$, respec- tively. The left sky-map is a flux map of UHECRs above $8 \mathrm{EeV}$ reported by Auger, indicating an obvious dipole structure of $6.5 \%$ amplitude with a $5.2 \sigma$ significance [11]. The direction of maximum amplitude is $125^{\circ}$ away from the galactic center, indicating an extragalactic origin for these ultrahigh-energy particles. An enhancement of the dipole amplitude above $4 \mathrm{EeV}$ is also reported [21]. This dipole structure and its amplitude are consistent with expectation if we assume the deflection of charged nuclei under the typical strength of the magnetic fields within our galaxy [22].

The central sky-map is a significance map of UHECR arrival directions above $57 \mathrm{EeV}$ observed with both TA and Auger [13]. The maximum excess appears as a "hotspot" centered at a right ascension of $147^{\circ}$ and a declination of $43^{\circ}$ with a $3.4 \sigma$ significance [12]. In addition, arrival directions of UHECRs are found to correlate with nearby extragalactic objects at a modest $2 \sim 3 \sigma$ significance level [23, 24]. There seems to be no measured excess in the direction of the Virgo cluster. Recently, Auger reported a $4.0 \sigma$ correlation between the positions of nearby starburst galaxies and the arrival directions of $9.7 \%$ of their measured UHECR events above $39 \mathrm{EeV}$ [25]. The remaining $90.3 \%$ of their measured events are consistent with an isotropic distribution. The right sky-map shows the arrival directions of UHECRs above $100 \mathrm{EeV}$. Curiously, two doublets (events having arrival directions within their corresponding uncertainties) can be seen, having a chance probability of $2.8 \sigma$ [14].

Except for the Auger dipole result above $8 \mathrm{EeV}$, these inconclusive results above $57 \mathrm{EeV}$ are limited by statistics at the highest energies due to the flux suppression. To further advance and establish the field of charged particle astronomy, a future ground array will require an unprece- 


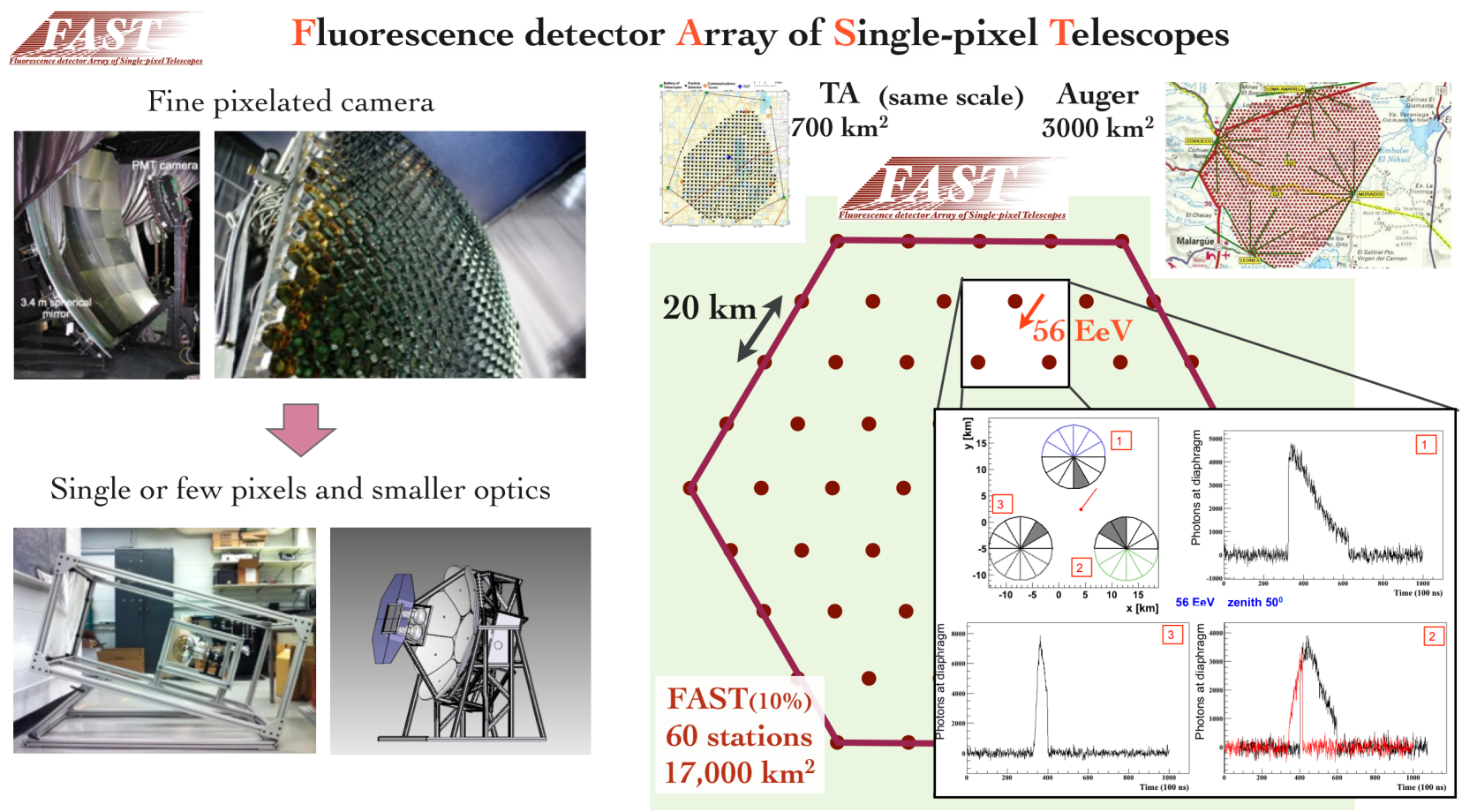

Figure 2. Schematic view of the Fluorescence detector Array of Single-pixel Telescopes: one of the possible solutions for a future giant ground array [18]. As a reference, the ground coverage of TA and Auger make up only $10 \%$ of the ground coverage of a full-scale array of FAST detectors. The expected signal emitted from a UHECR with an energy of $57 \mathrm{EeV}$ is shown in a simulation.

dented aperture, which is larger by an order of magnitude, and mass composition sensitivity above $100 \mathrm{EeV}$. In order to cover this enormous target volume, future detectors should be low-cost and easily-deployable. Worldwide collaboration is essential in successfully building such a giant ground array.

\section{Fluorescence detector Array of Single-pixel Telescopes (FAST)}

One of the possible solutions capable of fulfilling these requirements is a ground-based fluorescence detector array that is low-cost, easily-deployable, and has an unprecedented aperture which is an order of magnitude larger than that of Auger and TA $\times 4$.

The Fluorescence detector Array of Single-pixel Telescopes (FAST) [https://www.fast-project.org] consists of compact FD telescopes featuring a smaller light collecting area and far fewer pixels than current generation FD designs, leading to a significant reduction in cost.

In the current FD design, a large mirror system of $\sim 3.5 \mathrm{~m}$ diameter reflects a $30^{\circ} \times 30^{\circ}$ patch of the sky onto a focal plane composed of several hundred photomultiplier tubes (PMTs). In the FAST design, the same field-of-view is covered by just four PMTs at the focal plane of a compact segmented mirror of $1.6 \mathrm{~m}$ in diameter [26].

A significant cost reduction is expected due to the compact design of FAST, with smaller light collecting optics, a smaller telescope housing, and a smaller number of PMTs and associated electronics. Each FAST station would consist of 12 telescopes, covering $360^{\circ}$ in azimuth and $30^{\circ}$ in elevation. Powered by solar panels and with wireless connection, they will be deployed in a triangular arrangement with a $20 \mathrm{~km}$ spacing.

Figure 2 shows a schematic view of FAST, and the expected signal from a UHECR shower with coincidence detections at three adjacent stations. An example of the ground coverage using 60 stations is also indicated with a comparison to Auger and TA coverages. To achieve an order of magnitude larger effective aperture than Auger and $\mathrm{TA} \times 4,500$ stations are required after accounting for the standard FD duty-cycle. The operation of a full-size FAST array will provide conclusive results on the origin and acceleration mechanism toward $100 \mathrm{EeV}$, and open the window to charged-particle astronomy using UHECRs.

\subsection{Progress of developments on the FAST prototypes}

A first test of the FAST concept was performed in 2014 using a single $200 \mathrm{~mm}$ PMT at the focus of a $1 \mathrm{~m}^{2}$ Fresnel lens system at the Telescope Array site. Using the first prototype we detected 16 highly significant UHECR shower signals, and demonstrated excellent operational stability under conditions typical of field deployment [18].

Motivated by these encouraging results, we have developed and installed three full-scale FAST prototypes. The prototype, shown in Figure 3, consists of a segmented spherical mirror of $1.6 \mathrm{~m}$ diameter (produced at the Joint Laboratory of Optics in Olomouc, Czech Republic), and a UV band-pass filter (ZWB3, Shijiazhuang Zeyuan Optics) with a $1 \mathrm{~m}^{2}$ aperture. Four $200 \mathrm{~mm}$ PMTs (mod. 

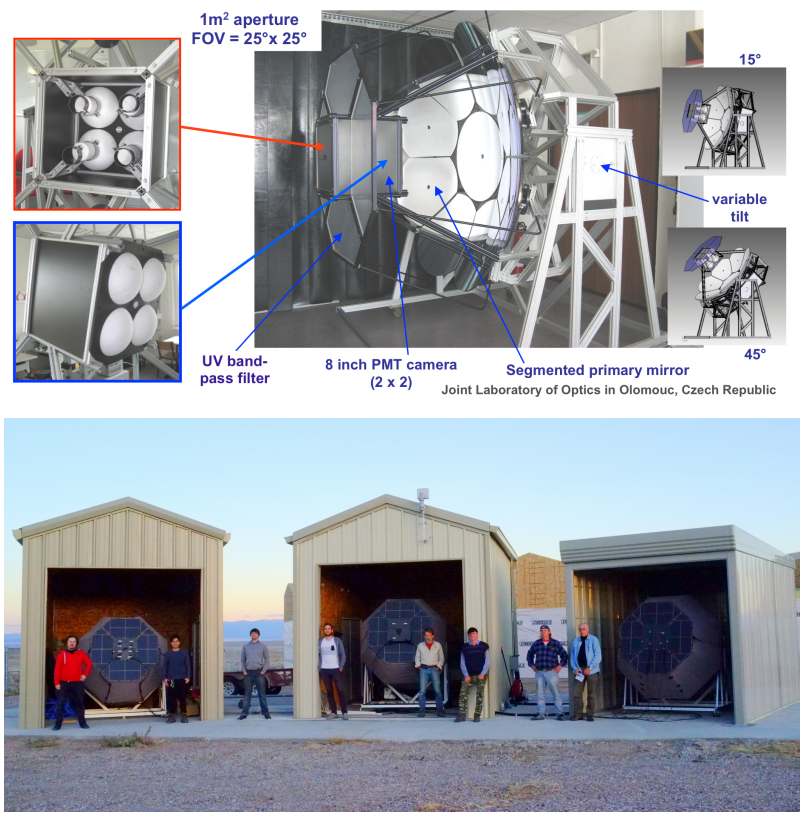

Figure 3. The full-scale FAST prototype developed at the Joint Laboratory of Optics in Olomouc, Czech Republic and three FAST prototypes installed at Black Rock Mesa site of Telescope Array Experiment.

R5912-03, Hamamatsu) and active bases (mod. E769401, Hamamatsu) are installed at the focal plane of the segmented mirror in a $2 \times 2$ matrix, covering a $30^{\circ} \times 30^{\circ}$ field of view. The telescope frame is covered with a shroud to shield the optical system from dust and stray light.

\subsection{Installation of three full-scale FAST prototypes at the Telescope Array site}

In October 2016, September 2017, and October 2018, three full-scale FAST prototypes were installed at the Telescope Array site as shown in Figure 3. The combined field of view of the prototypes covers $30^{\circ}$ in elevation and $90^{\circ}$ in azimuth. The telescope frames were assembled on site, before the PMTs were mounted in the camera box and the UV band-pass filter was installed at the telescope aperture. The telescopes were aligned astrometrically using a camera mounted to the exterior of the frame [27]. Following their installation, stable operation of the prototypes began via a remote connection, utilizing external air shower triggers from the adjacent TA fluorescence detector.

\subsection{Data-taking system of the prototypes}

High voltage is supplied to the four PMTs, which were calibrated in the laboratory [28] to a nominal gain of $5 \times 10^{4}$, by a high-voltage power supply (mod. N1470, CAEN). The PMT signals are amplified by a factor of 50 using a fast amplifier (mod. 777, Phillips Scientific), and passed through a $15 \mathrm{MHz}$ low-pass filter before digitization by a 14-bit FADC (mod. SIS3316, Struck Innovative Systeme) at a $50 \mathrm{MHz}$ sampling rate. The digitizer is hosted in a portable VME crate (mod. VME8008B, CAEN), together with a controller (mod. V7768, GE Intelligent platforms) and a GPS unit (mod. GPS2092, Hytec) which provides event time stamps. When a fluorescence telescope in the adjacent TA building is triggered by a candidate UHECR shower, an external trigger is issued to the FAST DAQ with a typical rate of $\sim 3 \mathrm{~Hz}$.

\subsection{Night-sky online monitoring systems}

The FAST all sky camera (FASCam) and sky quality monitor (SQM) are two instruments attached to the first FAST telescope building for monitoring of the night-sky background and cloud coverage. The FASCam and SQM are fully automated systems designed to measure the cloud coverage above the detector. Figure 4 shows measurements taken using these instruments as a function of time during a single night of observation. The "star visible ratio" quantifies the number of observed stars relative to the number of expected stars as given by a star catalog. The expected correlation between the "star visible ratio" and the night sky brightness as measured by the SQM can be seen.

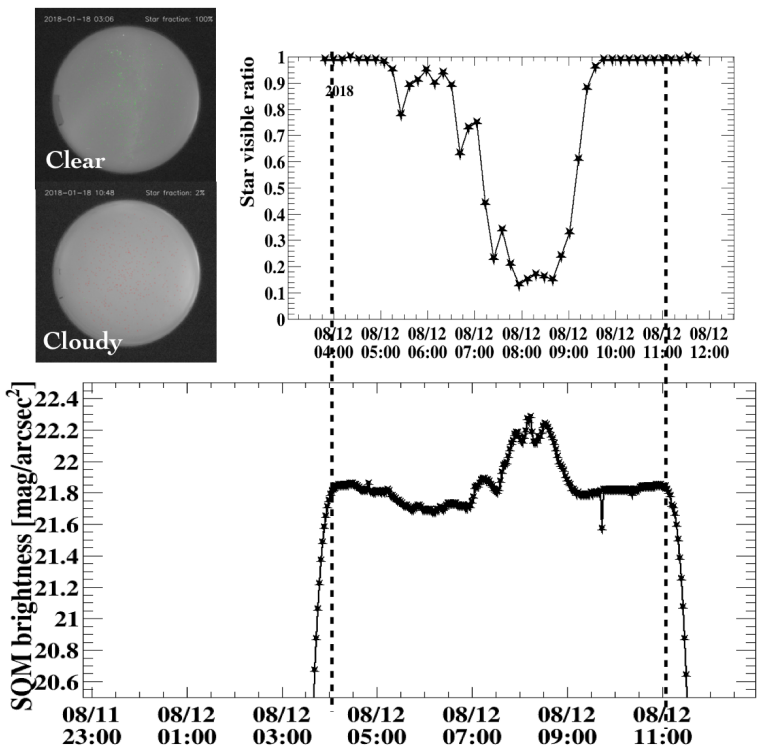

Figure 4. Example of images taken on clear and cloudy nights. The "star visible ratio" measured by the FAST all sky camera (FASCam) and the brightness observed by the sky quality monitor $(\mathrm{SQM})$ as a function of the observation time are shown.

\section{Preliminary results using three FAST prototypes}

One of the three FAST prototypes observes a half-hourly vertical laser signal at a distance of $21 \mathrm{~km}$. The laser signal is useful for monitoring the atmospheric transparency above the detector, which is one of the largest systematic uncertainties in the analysis of data collected using the fluorescence technique. Figure 5 shows a preliminary average trace of this vertical laser signal compared with the 
simulated expectation given a number of different atmospheric transparencies. In this example case, the measured trace is consistent with an atmosphere with a high aerosol loading.

By October 2018, the total observation time of the FAST prototypes is 425 hours. By searching for coincidences with the showers reconstructed by the Telescope Array fluorescence detectors [29], cosmic ray air showers with energies above $10^{19} \mathrm{eV}$ are selected. A top-down reconstruction algorithm is implemented to determine the best-fix shower parameters by comparing the measured signal trace to a library of simulated templates. Figure 6 shows a comparison of the preliminary waveforms for the highest energy cosmic-ray event observed with the FAST prototypes. The preliminary reconstructed energy is $\sim 2 \times$ $10^{19} \mathrm{eV}$. The simulated waveforms corresponding to these parameters show reasonable agreement with the data, although further understanding of the telescope calibration factors are required to reduce the discrepancy.

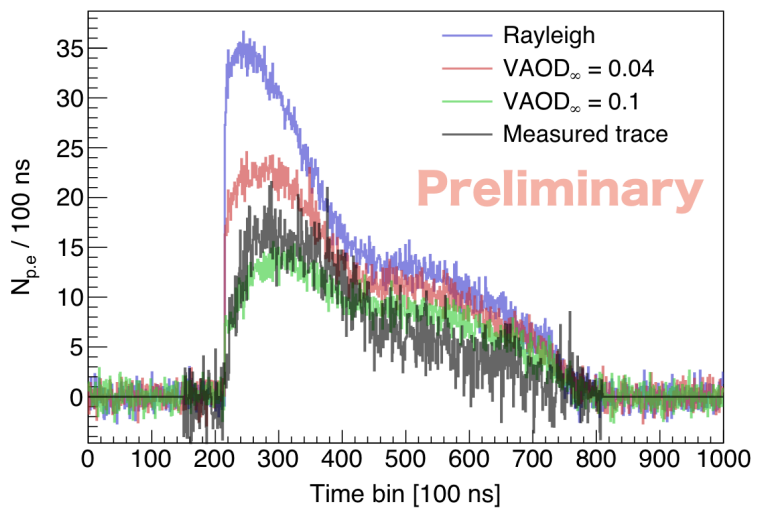

Figure 5. The measured trace (black line) from a distant vertical UV laser (284 shots averaged) at a distance of $21 \mathrm{~km}$ compared to the expected signals assuming various atmospheric transparencies: pure Rayleigh scattering (blue), vertical aerosol optical depth $(\mathrm{VAOD})=0.04$ (red), and 0.1 (green)

\subsection{Possible FAST applications}

We plan to install an additional identical FAST prototype at the Pierre Auger Observatory in 2019. This identical FAST prototype will allow for a cross-calibration of the energy and $X_{\max }$ scales of the Telescope Array and the Pierre Auger Observatory, as well as a comparison between the atmospheric transparency at both sites, an important source of systematic uncertainty in the fluorescence technique.

\section{Summary}

Measurements of UHECRs made by the Telescope Array Experiment and the Pierre Auger Observatory have firmly confirmed the suppression of the energy spectrum at the highest energies, and uncovered intriguing large-scale anisotropies in the arrival directions of UHECRs. A gradual transition to a heavier composition with increasing energy and a lack of desired small-scale anisotropies have also been observed.

In order to achieve a future $30,000 \mathrm{~km}^{2}$ effective ground coverage, we have developed a low-cost, easilydeployable fluorescence detector optimized for the detections of the highest energy cosmic rays. Three FAST prototypes have been installed at the Telescope Array site. We have analyzed vertical laser signals to investigate the atmospheric transparency above the detector, and measured UHECRs with energies above $10^{19} \mathrm{eV}$. We will continue the steady remote operation in order to further increase statistics.

Furthermore, we plan to install an identical FAST prototype at the Pierre Auger Observatory in order to perform a cross-calibration of the TA and Auger energy and $X_{\max }$ scales.

\section{Acknowledgements}

This work was supported by JSPS KAKENHI Grant Number 18H01225, 15H05443, and Grant-in-Aid for JSPS Research Fellow 16J04564 and JSPS Fellowships H25-339, H28-4564. This work was partially carried out by the joint research program of the Institute for Cosmic Ray Research (ICRR) at the University of Tokyo. This work was supported in part by NSF grant PHY-1713764, PHY-1412261 and by the Kavli Institute for Cosmological Physics at the University of Chicago through grant NSF PHY-1125897 and an endowment from the Kavli Foundation and its founder Fred Kavli. The Czech authors gratefully acknowledge the support of the Ministry of Education, Youth and Sports of the Czech Republic project No. LG15014, LE13012, LO1305.

\section{References}

[1] A.M. Hillas, Ann. Rev. Astron. Astrophys. 22, 425 (1984)

[2] J. Linsley, Phys. Rev. Lett. 10, 146 (1963)

[3] K. Greisen, Phys. Rev. Lett. 16, 748 (1966)

[4] G. Zatsepin, V. Kuzmin, JETP Lett. 4, 78 (1966)

[5] I. Valino (Pierre Auger Collaboration), PoS ICRC2015, 271 (2016)

[6] D. Ivanov, PoS ICRC2015, 349 (2016)

[7] V. Verzi, D. Ivanov, Y. Tsunesada, PTEP 2017, 12A103 (2017), 1705.09111

[8] A. Aab et al. (Pierre Auger Collaboration), Phys.Rev. D90, 122005 (2014), 1409.4809

[9] A. Aab et al. (Pierre Auger Collaboration), Phys.Rev. D90, 122006 (2014), 1409. 5083

[10] A. Aab et al. (Pierre Auger Collaboration), Phys. Rev. D96, 122003 (2017), 1710.07249

[11] A. Aab et al. (Pierre Auger Collaboration), Science 357, 1266 (2017), 1709.07321

[12] R. Abbasi et al. (Telescope Array Collaboration), Astrophys.J. 790, L21 (2014), 1404 . 5890 

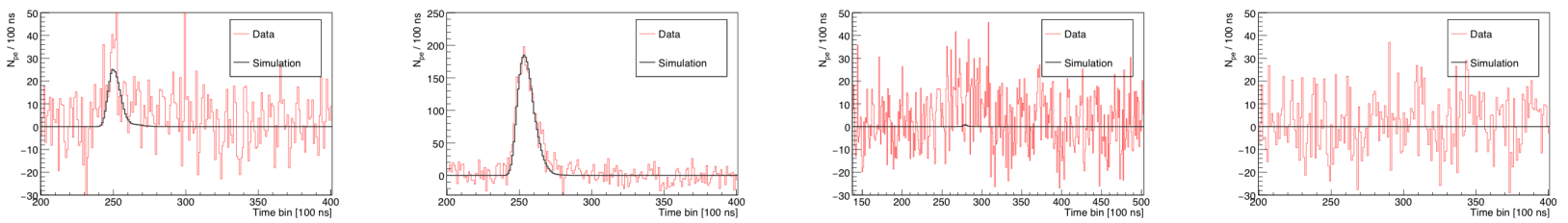

\section{Preliminary}
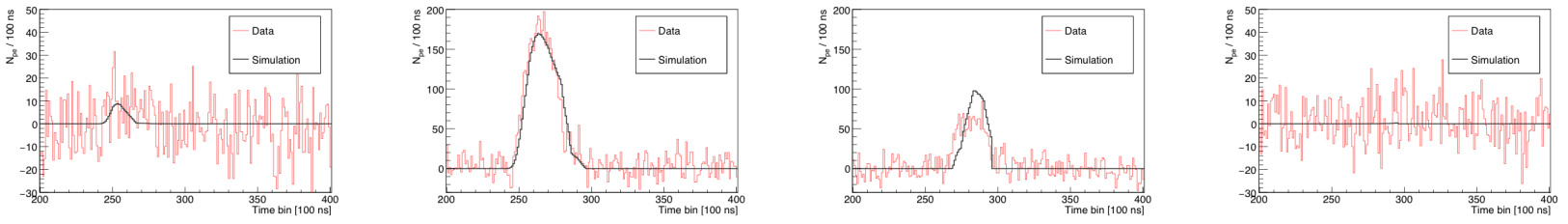

Figure 6. The waveforms of the highest energy cosmic-ray event observed with the FAST prototypes. The red histograms show the recorded data and the black curves indicate the best-fit signal from a top-down reconstruction.

[13] K. Kawata et al. (Telescope Array Collaboration), PoS ICRC2015, 276 (2016)

[14] S. Troitsky et al. (Telescope Array Collaboration), PoS ICRC2017, 548 (2018)

[15] A. Aab et al. (Pierre Auger Collaboration), Nucl. Instrum. Meth. A798, 172 (2015), 1502.01323

[16] H. Tokuno, Y. Tameda, M. Takeda, K. Kadota, D. Ikeda et al., Nucl. Instrum. Meth. A676, 54 (2012), 1201.0002

[17] T. Abu-Zayyad et al. (Telescope Array Collaboration), Nucl. Instrum. Meth. A689, 87 (2012), 1201.4964

[18] T. Fujii et al., Astropart. Phys. 74, 64 (2016), 1504.00692

[19] E. Barcikowski et al. (Pierre Auger, Yakutsk Collaborations), EPJ Web Conf. 53, 01006 (2013), 1306.4430

[20] V. de Souza (Pierre Auger and Telescope Array Collaborations), PoS ICRC2017, 522 (2018)
[21] A. Aab et al. (Pierre Auger), Astrophys. J. 868, 4 (2018), 1808.03579

[22] A. di Matteo, P. Tinyakov, Mon. Not. Roy. Astron. Soc. 476, 715 (2018), 1706.02534

[23] T. Abu-Zayyad et al. (Telescope Array Collaboration), Astrophys.J. 757, 26 (2012), 1205. 5984

[24] P. Abreu et al. (Pierre Auger Collaboration), Astropart.Phys. 34, 314 (2010), 1009. 1855

[25] A. Aab et al. (Pierre Auger Collaboration), Astrophys. J. 853, L29 (2018), 1801.06160

[26] D. Mandat et al. (FAST Collaboration), JINST 12, T07001 (2017)

[27] D. Mandat, M. Palatka, M. Pech, P. Schovanek, P. Travnicek et al., PoS ICRC2017, 389 (2017)

[28] M. Ave et al. (AIRFLY Collaboration), Astropart.Phys. 42, 90 (2013), 1210.6734

[29] R.U. Abbasi et al. (Telescope Array Collaboration), Astropart. Phys. 80, 131 (2016), 1511.07510 